\title{
Öffentlichkeitsbeteiligung bei Infrastrukturprojekten als Herausforderung für Politik und Verwaltung
}

\section{Lars Rademacher, Klaus Lintemeier und Heiko Kretschmer}

Mehr als $80 \%$ der Deutschen wünschen sich mehr Mitsprachemöglichkeiten in politischen Entscheidungsprozessen. Zivilgesellschaftliche Initiativen auf lokaler Ebene, Bürgerbewegungen und der steigende Einfluss von Nichtregierungsorganisationen (NGO) sind nur einige Beispiele für den immer deutlicher wahrnehmbaren Wunsch, gesellschaftliche Entwicklungen aktiv mitzugestalten. Mittlerweile sind in Publikationen der Bertelsmann Stiftung (2014) mehr als hundert Bürgerbeteiligungsformate verzeichnet.

Den Kern vieler dieser Verfahren bilden dialogorientierte und indirekt angelegte Beteiligungsverfahren, die einer ,Wiederentdeckung von Formen der öffentlichen Debatte über Angelegenheiten des Gemeinwesens" (Geißel et al. 2014, S. 16) gleichkommen und im Wesentlichen der Vorbereitung von Entscheidungen dienen. Dem stehen Verfahren gegenüber, die direktdemokratische Entscheidungen über Sachfragen ermöglichen sollen und dafür konkrete, meist elektronische Abstimmungsverfahren benötigen. Während bei der dialogorientierten Variante Öffentlichkeit und Bürgergruppen lediglich gehört und in den Entscheidungsprozess vorbereitend einbezogen werden, entscheiden sie bei direktdemokratischen Formen mit (Geißel et al. 2014).

L. Rademacher $(\bowtie)$

Fachbereich Media, Hochschule Darmstadt, Darmstadt, Deutschland

E-Mail: lars.rademacher@h-da.de

K. Lintemeier

Lintemeier Advisors, München, Deutschland

E-Mail: klaus.lintemeier@lintemeier.de

H. Kretschmer

Johannsen + Kretschmer Strategische Kommunikation GmbH, Berlin, Deutschland

E-Mail: h.kretschmer@jk-kom.de 


\section{Gestiegene Komplexitäten}

Die Rahmenbedingungen von Industrie- und Infrastrukturprojekten sind komplex: Auf der einen Seite eine Öffentlichkeit, die verstärkt Dialog- und Beteiligungsformen jenseits der formalen Beteiligungsverfahren einfordert, auf der anderen Seite eine Verwaltung mit verschiedenen Fachbereichen und Zuständigkeiten sowie bindenden verwaltungs- und umweltrechtlichen Vorgaben. Damit stellt sich die Frage, wie Projekte heute erfolgreich umgesetzt werden können.

Die zentralen Erfolgsfaktoren für Vorhabenträger sind einerseits eine schnelle und rechtssichere Bearbeitung ihrer Projekte durch die Verwaltung und andererseits eine Öffentlichkeit, die ihre Vorhaben trägt. Beides ist heute jedoch nicht garantiert. Verwaltungsprozesse stecken in Zielkonflikten zwischen Politik, Recht und der internen Zuständigkeitslogik fest. Die öffentliche Meinung wird nicht selten von kritischen NGOs geprägt, die zunehmend medial präsent und vernetzt sind.

Der Bundesgesetzgeber hat auf die neuen Herausforderungen reagiert und mit der Neuregelung des $§ 25$ Abs. 3 Verwaltungsverfahrensgesetz (VwVfG) vom 7. Juni 2013 das Instrument der frühen Öffentlichkeitsbeteiligung eingeführt. Dieses Gesetz wurde mittlerweile auch in den Bundesländern umgesetzt. Zugleich sind weitere Standards und Richtlinien entstanden, so etwa der Planungsleitfaden für Infrastrukturprojekte des Landes Baden-Württemberg oder die Richtlinie VDI 7000 des Vereins Deutscher Ingenieure. Doch damit ist die Frage, wie Beteiligung und Dialog erfolgreich in die Planung von Projekten und Vorhaben integriert werden können, nicht beantwortet.

Die meisten Beteiligungsverfahren sollen zu akzeptierten Lösungen führen, sehen sich aber zunehmend mit einer Frontalopposition konfrontiert. Demokratietheoretische Ansätze verlieren hier schnell ihre Wirkungsmacht (Crouch 2008). Die Perspektive der Vorhabenträger und die rechtlichen Rahmenbedingungen werden im Partizipationsdiskurs bisher kaum berücksichtigt.

Des Weiteren befindet sich unsere Gesellschaft in einem radikalen Wandel. Aus kommunikativer Sicht fällt dabei vor allem eines auf: Das Zusammenspiel zwischen Organisationen und ihren Anspruchsgruppen (Stakeholdern) verändert sich. Vormals wichtige Stakeholder verlieren an Bedeutung oder verschwinden, bisher wenig beachtete Gruppen werden wichtiger und neue kommen hinzu (Lintemeier und Rademacher 2013). Auch die von ihnen formulierten Ansprüche und Erwartungen gegenüber Organisationen - seien es Unternehmen, wissenschaftliche oder politische Institutionen - verändern sich. Grund dafür sind nicht zuletzt gesellschaftliche Entwicklungen wie der Wertewandel oder die Digitalisierung: 
Dank der sozialen Medien kann jeder heute jederzeit und überall Inhalte mit bisher ungeahnten Reichweiten und innerhalb kürzester Zeit weltweit verbreiten. Im Zuge dieser neuen Möglichkeiten erodiert die Rolle von Journalisten als Gatekeeper. Zugleich erhöht sich das Tempo: wer im Netz nicht innerhalb von $24 \mathrm{~h}$ reagiert, hat die Deutungshoheit über seine Themen schnell verloren. Die neuen Medien forcieren zudem eine neue Kultur der Partizipation: Bürger fordern zunehmend mehr Teilhabe- und Beteiligungsmöglichkeiten (zum Beispiel im Rahmen von Infrastrukturprojekten). Von Unternehmen und Organisationen wird zunehmend mehr Transparenz und Kooperation auf Augenhöhe gefordert (Heller 2018). So wandeln sich beispielsweise Kunden zu selbstbewussten Prosumern, die aktiv auf Produktentwicklungen Einfluss nehmen.

Die Bedeutung der Stakeholder für Organisationen nimmt also zu - und damit auch der Druck auf Organisationen. Denn entziehen die relevanten Anspruchsgruppen einem Unternehmen ihr Vertrauen, kann das zu einem echten Geschäftsrisiko werden. Stabile und gute Stakeholder-Beziehungen sind heute das A und $\mathrm{O}$ für einen langfristigen Geschäftserfolg. Das führt zu drei fundamentalen Veränderungen, die wir nachfolgend darstellen.

\section{Veränderte Beteiligungskonstellationen}

\subsection{Frühzeitige Einbindung als Pflicht}

Politische Entscheidungen im ,stillen Kämmerlein“ fällen oder Großprojekte ohne Einbindung der Anspruchsgruppen realisieren - das kann einem heute teuer zu stehen kommen, wie das Bahnhofsprojekt Stuttgart 21 exemplarisch zeigt. Die Menschen wollen mitreden, vor allem wenn geplante Vorhaben ihr Leben mehr oder minder direkt betreffen. Wie eingangs erwähnt, wünschen sich einer Studie der Bertelsmann Stiftung (2014) zufolge mehr als $80 \%$ der Bürger mehr Mitsprachemöglichkeiten und immer weniger lassen sich von einem Behördenstempel beeindrucken. Das zeigt der Protest gegen bereits genehmigte Verfahren und Projekte.

Die Gründe für das neue Bedürfnis nach Beteiligung sind vielfältig. Sie reichen von persönlichen Interessen über das sogenannte Sankt-Florians-Prinzip (neudeutsch: Nimby) und fehlendem Verständnis bis hin zu Entfremdung und Misstrauen. Zugleich aber lässt sich ein Phänomen beobachten, das als Beteiligungsparadoxon bezeichnet wird: Am Anfang eines Verfahrens sind die Spielräume für Beteiligung und Mitgestaltung groß und das Interesse der Öffentlichkeit klein (es ist ja auch noch nichts zu spüren). Es nimmt parallel zum 
Projektfortschritt in dem Maße zu, wie das Vorhaben spürbar wird (Rademacher und Lintemeier 2015). Doch je konkreter das Vorhaben wird, desto kleiner werden die Möglichkeiten der Einflussnahme seitens der Öffentlichkeit. Das führt zu Blockaden. Aufgabe der Politik ist es, die Öffentlichkeit über ihre Beteiligungsmöglichkeiten zu informieren und auf ihre möglichst frühe Einbindung bei den Vorhabenträgern hinzuwirken. Diese stehen damit vor der Herausforderung, das Interesse der Bürger zu wecken und einen konstruktiven Dialog über ein faktisches ,Nichts` zu initiieren, das erst entstehen muss. Wie kann das gelingen?

\subsection{Veränderungen in der Meinungsbildung}

Sie fördern den öffentlichen Diskurs und die Meinungsbildung, das Wissen und das Problembewusstsein. Der Systemtheoretiker Niklas Luhmann (1996) sah sie daher als ,Fenster zur Welt‘. Für andere sind sie Garant einer pluralistischen demokratischen Gesellschaft. Gemeint sind die klassischen Medien: Presse, Rundfunk und Fernsehen. Mit dem Aufkommen des ,Social Web“ haben sich ihre Rolle und ihre Bedeutung jedoch dramatisch verändert.

Facebook, YouTube, Twitter \& Co. laufen klassischen Medienformaten den Rang ab. Online-Publisher werden zu einflussreichen Meinungsmachern (neudeutsch: Influencern) und konterkarieren den journalistischen Status des ,Torhüters zum Wissen'. Damit verändert sich auch der Prozess der Meinungsbildung. Sie wird nicht nur digitaler, sondern auch direkter, dezentraler und dialogischer. Neben die klassischen Meinungsbildner Politik und Medien treten vermehrt neue Influencer mit klaren Positionen und überzeugenden Argumentationen. Als professionelle Social-Media-Manager wissen sie Themen zu lancieren und zu besetzen.

Hier tun sich vor allem kritische NGOs, Bürgerinitiativen und Interessengruppen hervor und stellen Politik wie Unternehmen damit vor neue Herausforderungen: ,Herr seiner Themen“ ist heute nur derjenige, der 1) schnell kommuniziert und 2) das Vertrauen der Öffentlichkeit genießt. Beides trifft weder auf Politik noch auf Unternehmen zu. „Die Menschen [fühlen] sich zunehmend entfremdet von den Verantwortungsträgern des Gemeinwesens", heißt es in der RWE-Akzeptanzstudie (2012, S. 18). „Ihnen ist das Vertrauen abhandengekommen, sowohl in die Politik als auch in große Unternehmen“ (RWE 2012, S. 18). Damit stellt sich die Frage, wie die traditionellen Institutionen - allen voran Unternehmen - die Themenführerschaft zurückerlangen und sich als glaubwürdige Akteure Gehör verschaffen können. 


\subsection{Zwischen Dialogkultur und Prozessstandardisierung}

Als Reaktion auf das neue Beteiligungsbedürfnis suchen Politik und Wirtschaft nach neuen Wegen der politischen Steuerung und Kommunikation. Während Politiker eine neue Dialogkultur zu etablieren versuchen, treiben Behörden, Verbände und Unternehmen die Standardisierung und Professionalisierung von Beteiligung in Bau- und Infrastrukturvorhaben massiv voran. Dafür gibt es eine Menge guter Beispiele:

- Das Bundesministerium für Verkehr, Bau und Stadtentwicklung hat bereits 2012 ein Handbuch für gute Bürgerbeteiligung veröffentlicht, das parallel zum 2013 in Kraft getretenen „Gesetz zur Verbesserung der Öffentlichkeitsbeteiligung und Vereinfachung von Planfeststellungsverfahren" entwickelt wurde.

- Der Verein Deutscher Ingenieure (VDI) hat nach der Richtlinie VDI 7000 zur „Frühen Öffentlichkeitsbeteiligung bei Industrie- und Infrastrukturprojekten“" einen Leitfaden zur Integration von Öffentlichkeitsbeteiligung in das Projektmanagement von Infrastruktur- und Industrieprojekten entwickelt (Verein deutscher Ingenieure e. V. 2014).

- Die Landesregierung von Baden-Württemberg hat 2013 eine verbindliche Verwaltungsvorschrift zur Intensivierung der Öffentlichkeitsbeteiligung in Planungs- und Zulassungsverfahren erarbeitet, die 2014 in Kraft getreten ist und in ihrer rechtlichen Tragweite bundesweit einmalig ist (Staatsministerium Baden-Württemberg 2014).

Unternehmen und Vorhabenträger haben eigene Standards und Leitfäden für Öffentlichkeitsbeteiligung entwickelt. All dies sind wichtige Schritte in die richtige Richtung. Doch die Frage, wie Dialog und Beteiligung erfolgreich in die unternehmerische Praxis übersetzt werden können, ist damit längst noch nicht beantwortet. Unter Berücksichtigung branchen- und projektspezifischer sowie gesetzlicher Rahmenbedingungen werden Ansätze entwickelt, wie Dialog und Beteiligung in unternehmerische Prozesse und Verfahren integriert werden können.

In der Arbeitswelt würden Neinsager keinen Orden bekommen, so Karrierecoach Martin Wehrle (2015) im Interview mit ZEIT ONLINE. In der Öffentlichkeitswelt dagegen schon. Es ist deutlich einfacher, gegen etwas als für etwas zu sein. Wer für etwas ist, macht sich schnell verdächtig: Wurde sie ,gekauft'? Ist er naiv? Wurden sie instrumentalisiert? Niemand möchte unter diesem Verdacht stehen und so wundert es nicht, dass Protest in der Regel laut und Fürsprache leise ist. 
Bei wichtigen politischen Entscheidungen oder in Genehmigungsverfahren kann die ,Stille der Fürsprecher' jedoch zum echten Risiko werden. Ohne Gegenstimmen kann die Proteststimmung auf die allgemeine Stimmungslage überspringen und eine Initiative, wenn nicht stoppen, so doch blockieren. Der Bürgerentscheid gegen die Teilbebauung des ehemaligen Tempelhofer Flughafens wäre vermutlich weniger erfolgreich gewesen, hätte die Initiative „100\% Tempelhofer Feld“ die öffentliche Meinung nicht einseitig dominiert. Damit stellt sich die Frage: Wie können die Fürsprecher mobilisiert werden? Und mit Blick auf die Gegenseite fragt sich: Wie geht man mit kritischen Stakeholdern um, vor allem dann, wenn es sich dabei um eine Frontalopposition handelt?

\section{$3 \quad$ Partizipation online gestalten}

Mit der zunehmenden Digitalisierung verbinden Online-Beteiligungen das gesteigerte öffentliche Interesse an unterschiedlichen Vorhaben sowie den Wunsch der Bevölkerung, früh und umfassend in Entscheidungsprozesse eingebunden $\mathrm{zu}$ werden. Dies betrifft sowohl große Infrastrukturvorhaben mit formellen Beteiligungsverfahren als auch gesamtstädtische bis kleinteilige Stadtentwicklungsprozesse mit informellem Charakter. Doch auch wenn sich nach und nach Ernüchterung über die Möglichkeiten von Online-Dialogen breit macht, werden sie nach wie vor angewandt - wenn auch zu großen Teilen ineffektiv. Häufig wird digitale Beteiligung als ,Allheilmittel ‘ betrachtet, dass Akzeptanz für Vorhaben schaffen soll und Repräsentationsprobleme lösen kann. Dabei gilt oftmals das Motto ,Methode vor Inhalt" und Vorhabenträger stürzen sich in Beteiligungsprozesse im Internet, ohne sich darüber im Klaren zu sein, was tatsächlich digital funktioniert und was nicht. Dann werden zwar Beteiligungsfenster eröffnet, aber es mangelt an klaren Zielen, konkreten Fragestellungen und einer projektspezifischen Steuerung - was essenziell für eine erfolgreiche Einbindung von Stakeholdern im Netz ist.

Eine systematische Analyse der Möglichkeiten und Grenzen aus Sicht der Vorhabenträger stand bislang aus. Es ist empfehlenswert, die bisherigen Erfahrungen zur Online-Beteiligung auszuwerten, um darauf aufbauend Handlungsempfehlungen zu entwickeln, die Projektverantwortliche bei der erfolgreichen Umsetzung von Beteiligungsverfahren unterstützen sollen. Zur Systematisierung wurden drei Ebenen der Online-Beteiligung identifiziert: 1) Online-Formate als reine Informations- und Dokumentationsplattformen, 2) Online-Formate als Plattformen für konkrete Teilhabe und 3) Debatten in öffentlichen Online-Foren. 
Diese Aussagen treffen teilweise auch für Offline-Beteiligungsformate zu, haben aber für digitale Partizipation eine besondere Bedeutung. So spielt die Moderation von Online-Beteiligungsprozessen beispielsweise eine geringere Rolle als bei Offline-Formaten. Hingegen ist der Umgang mit Echoräumen eine besondere Herausforderung im Netz.

\subsection{Akzeptanz-Management lässt sich nicht ins Netz auslagern}

Viele Projektverantwortliche erwarten durch Online-Beteiligung einen Akzeptanzschub für ihr Vorhaben. Bisherige Erfahrungen zeigen allerdings, dass Online-Verfahren bei den Bürgern auf kaum messbare Akzeptanz stoßen. E-Partizipation kann zwar eine konstruktive Ergänzung für Offline-Beteiligungsverfahren sein, jedoch keinesfalls einen Ersatz darstellen. Es gelingt Online-Formaten nicht, Involvement aufseiten der User herzustellen, was eine konstruktive Beteiligung an einem Diskurs verhindert und eine Akzeptanzschaffung - sowohl für das Verfahren als auch für das Projekt - unmöglich macht. Aber: Vorhabenträger können im Internet ihre Projekte auch gezielt emotional bewerben und neben der Bereitstellung von Fakten und Argumenten einer Community aus Fürsprechern ein Forum bieten.

\subsection{Beteiligungsinteresse im Internet muss aktiv hergestellt werden}

Das Potenzial digitaler Beteiligung, grundsätzlich alle relevanten Stakeholder zu erreichen, darf nicht mit ihrer Aktivierung gleichgesetzt werden. Nicht alle Bevölkerungsgruppen lassen sich gleichermaßen über das Internet aktivieren und Verzerrungseffekte treten häufig auf. Zwar können mit Online-Formaten potenziell auch Nutzer erreicht werden, die über klassische Beteiligungsformate nicht angesprochen werden. Dass diese jedoch von selbst auf Vorhabenträger zugehen, ist ein Trugschluss und offenbar einer der Hauptgründe für die niedrigen Teilnehmerzahlen bei Online-Dialogen. Viele Beteiligungsportale werden von Medien kaum wahrgenommen und erfahren dementsprechend eine geringe Aufmerksamkeit. Daher sollten Vorhabenträger ihre Online-Portale über diverse Medienkanäle offensiv bewerben und dabei auch mediale Räume betreten, in denen sich Nutzer in ihrem Alltag aufhalten und die nicht der Kontrolle der Projektverantwortlichen unterliegen. Wichtig ist in diesem Zusammenhang, dass 
das betreffende Beteiligungsverfahren im Internet durch die Projektverantwortlichen anmoderiert wird, sodass Qualitäten und Quantitäten des Verfahrens (im Sinne von Beteiligungsfenstern, Beteiligungszeiträume, Rollenverteilung etc.) für die Öffentlichkeit nachvollziehbar sind.

\subsection{Online-Beteiligung kann Verfahrenslegitimität steigern}

Digitale Beteiligung kann zur Legitimation (nicht zur Akzeptanz) von Projekten und Beteiligungsprozessen beitragen, da sie jedem Interessierten, unabhängig von Zeit und Ort, ermöglichen, sich einzubringen. Sie können im Wesentlichen zur Anwendung sämtlicher Beteiligungsstufen genutzt werden. Von der Information über die Konsultation bis hin zur aktiven Beteiligung lassen sich alle Vorgänge grundsätzlich auch ins Netz auslagern. Konstruktive Partizipation gelingt aber nur, wenn bei den beteiligten Stakeholdern bereits ein Interesse an einem gemeinsamen Diskurs zur Verbesserung des geplanten Projekts vorhanden ist. Dann können Online-Formate dazu genutzt werden, einen laufenden Dialog fachlich zu vertiefen und planungsrelevante Hinweise zu erhalten. Voraussetzung ist allerdings, dass es gelingt, die Ergebnisse der digitalen Beteiligung mit Offline-Formaten sinnvoll zu verknüpfen. Zudem können Vorhabenträger über Online-Verfahren Nutzer aktivieren, die das Vorhaben bzw. das Beteiligungsverfahren auf der Plattform unterstützen. Zu Projekt- bzw. Verfahrensbefürwortern zählen häufig Träger öffentlicher Belange. Aber auch Unternehmen oder Privatpersonen können von Vorhabenträgern explizit als Fürsprecher für das Online-Beteiligungsverfahren gewonnen werden, um dadurch die Verfahrenslegitimität zu erhöhen.

\subsection{Mit konkreten Fragestellungen brauchbare Ergebnisse generieren}

Für den Erfolg des Beteiligungsverfahrens ist eine eindeutige Fragestellung entscheidend. Gerade komplexe Themen müssen so weit vereinfacht werden, dass sie leicht zu verstehen sind und einen schnellen Zugang ermöglichen. Dabei müssen fallspezifische Entscheidungen getroffen werden, die von der jeweiligen Zielsetzung abhängig sind. Soll mit dem Dialog insbesondere die Verfahrenslegitimität gesteigert werden, gilt es möglichst viele Personen zu aktivieren (,Dia$\log$ in die Breite'). Je niedrigschwelliger die Fragestellung, umso unkonkreter 
werden zwar die Beiträge, jedoch können so mehr Antworten generiert werden. Allerdings muss darauf geachtet werden, dass die Fragestellung nicht den Eindruck erweckt, dass das Beteiligungsfenster größer ist als tatsächlich vorgesehen. Sollen durch das Verfahren hingegen planungsrelevante Hinweise generiert werden, muss ein ,Dialog in die Tiefe' gewählt werden. Komplexere Fragestellungen sind zu stellen, um dadurch konkrete Angaben zu erhalten. Dies wirkt unter Umständen abschreckend auf bestimmte Nutzergruppen und der Kreis der Beteiligten reduziert sich deutlich. Hilfsmittel (wie bspw. Karten) können herangezogen werden, um Fragestellungen weiter zu konkretisieren.

\subsection{Online-Formate brauchen Design und Usability}

Viele Beteiligungsplattformen im Internet scheinen sich rein auf Funktionalität zu konzentrieren, wodurch ein modernes und vor allem ansprechendes Design in den Hintergrund rückt. Dabei ist es wichtig, die Portale so zu gestalten, dass Nutzer sich dort gerne aufhalten und außerdem schnell und einfach zurechtfinden. Vorhabenträger sollten immer davon ausgehen, dass ein Nutzer zum ersten Mal an einer Online-Beteiligung teilnimmt und die Webseite dementsprechend aufbauen. Der Internetauftritt sollte dem Nutzer außerdem signalisieren, welchen Mehrwert er durch die Beteiligung erhält. Dazu müssen auch leicht anzuwendende Tools angeboten werden, die es ermöglichen, konkrete Hinweise zu geben bzw. zu erhalten. Soll eine breite Beteiligung der Öffentlichkeit zu einem Issue erreicht werden, ist der Zugang im Internet möglichst niedrigschwellig zu gestalten (d. h. beispielsweise keine Registrierung der Nutzer als Voraussetzung für die konkrete Teilhabe).

\subsection{Organisationsentwicklung als unternehmensinterner Erfolgsfaktor}

Unabhängig vom gewählten Format (online oder offline) sollten Vorhabenträger vor Beginn und Konzeption eines Beteiligungsprozesses klären, ob ihre Organisation ein lernendes, sich selbst hinterfragendes System ist. Denn zum einen gilt es, die aus einer Öffentlichkeitsbeteiligung resultierenden Ideen und Anregungen personell und technisch zu verarbeiten. Zum anderen sollte es Anreize geben, diese Impulse der Stakeholder im Unternehmen zu nutzen. Dazu gehören beispielsweise auch kritische Mitarbeiter innerhalb einer Institution, die bereit sind, Inputs aufzugreifen und ,Übersetzungsleistungen“ innerhalb des Unternehmens 
zu erbringen (d. h. beispielsweise planungsrelevante Hinweise der Öffentlichkeit in die unternehmensinterne Projektplanung zu integrieren), damit gemeinsam die beste Lösung erreicht werden kann.

\subsection{Online-Beteiligung ist kein Selbstläufer}

Um Stakeholder im Internet einzubinden, reicht es nicht aus, eine Online-Plattform bereitzustellen, auf der Nutzer Anmerkungen hinterlassen können. Dafür existieren zwar mittlerweile diverse Tools, deren Anwendung allein schafft allerdings noch kein durchdachtes Beteiligungskonzept. Bereits im Vorfeld müssen Vorhabenträger ein konkretes Ziel formulieren, das durch die Online-Beteiligung erreicht werden soll. An diesem Ziel sollte sich das Verfahren orientieren. Das Beteiligungsverfahren muss von Projekt zu Projekt überprüft und ggf. neu konzipiert werden, um letzten Endes der Zielerreichung zu dienen und nicht nur Traffic zu generieren oder als ,Internet-Leiche “ im Netz zu verstauben. ,Laufen lassen “ ist keine Option. Fortlaufende Erfolgskontrolle, Prozesssteuerung und ggf. Nachjustierung des Beteiligungsverfahrens sind essentiell.

\subsection{Mit klaren Spielregeln ans Ziel}

Vorhabenträger müssen Erwartungen der Stakeholder managen und Spielregeln transparent und eindeutig kommunizieren. In diesem Zusammenhang ist es auch relevant, eine klare Trennung politischer Debatten von der eigentlichen Projektkommunikation der Vorhabenträger zu gewährleisten. Sowohl die Möglichkeiten als auch die Grenzen des Online-Verfahrens müssen erkennbar sein. Zudem ist klar zu vermitteln, wie groß das Beteiligungsfenster ist. Insbesondere wenn es um konkrete Teilhabe geht, ist der Nutzer auf seine Beteiligungsspielräume und -begrenzungen aufmerksam zu machen. Es sollte vermieden werden, durch missverständliche Sprachregelungen Erwartungen zu wecken, die vom Verfahren nicht erfüllt werden können. Entscheidend ist, den beteiligten Bürgern ein Feedback zu geben, wie mit ihren Hinweisen im weiteren Prozess umgegangen wird. ,Rückkopplungsschleifen" tragen hierbei maßgeblich zu Transparenz und Verfahrenslegitimität bei. 


\section{$4 \quad$ Hindernisse früher Öffentlichkeitsbeteiligung}

Gerade im Bereich der großen Infrastrukturprojekte allerdings gehen die Erwartungshaltungen besonders deutlich auseinander respektive treffen direktdemokratische Beteiligungswünsche der Öffentlichkeit auf etablierte Beteiligungsverfahren in Politik und Verwaltung (Römmele und Schober 2013). In diesem Kontext spielt die frühe Öffentlichkeitsbeteiligung in den letzten Jahren eine zunehmende Rolle. Dies zeigen u. a. die oben erwähnten Fallbeispiele des Handbuchs für gute Bürgerbeteiligung des Bundesministeriums für Verkehr, Bau und Stadtentwicklung (Krappel und Freiherr von Süsskind-Schwendi 2012), sowie die Richtlinie VDI 7000 des Vereins Deutscher Ingenieure (VDI).

Darin geht der VDI über die gesetzlichen Vorgaben und Erwartungshaltungen hinaus, ,da sie (i. e. die Richtlinie) Öffentlichkeitsbeteiligung als integrierten Teil des Projektmanagements von Infrastruktur- und Industrieprojekten begreift", wie Joachim Schwab (2014, S. 284) betont. In Baden-Württemberg seien Landesbehörden bereits verpflichtet, dritte Vorhabenträger auf die VDI 7000 hinzuweisen. Allerdings komme ihr keine rechtliche Verbindlichkeit zum Vollzug zu; auch gebe sie nicht den "Stand der Beteiligungstechnik“ (S. 284) wieder, urteilt Schwab (2014) und empfiehlt den Behörden insgesamt Zurückhaltung: „Methodenberatung gehört weder zum behördlichen Auftrag, noch besitzen Behörden die dafür notwendige Kompetenz oder verfügen über die entsprechenden Ressourcen“ (Schwab 2014, S. 284).

Damit liegt der Ball aufseiten der Politik, die sich überlegen muss, wie viel Bürgerbeteiligung sie will und wünscht - einerseits um die bestehende Lethargie und Legitimationskrise zu überwinden, andererseits um den verbreiteten Beteiligungswünschen nachzukommen. Doch will sie das überhaupt? Wie weit möchten politische Entscheider sich dem Bürger öffnen, wie viel Öffentlichkeitsbeteiligung wünschen sie sich oder wollen sie zulassen? Und wer will am meisten Austausch mit dem Bürger? Landes- oder Kommunalpolitiker?

Das wollten wir von denen wissen, die besonders unmittelbar von Bauund Infrastrukturmaßnahmen betroffen sind - als Landtags- oder Kreistagsabgeordnete, als Ministerialbeamte, Landräte, Stadtverordnete oder Kommunalpolitiker. Im Rahmen eines Befragungsprojekts wurden über 2000 deutsche Politiker aller Entscheidungsebenen befragt ${ }^{1}$. 272 Teilnehmer haben an

\footnotetext{
${ }^{1}$ Unterstützt wurde die Befragung durch das Fachmagazin politik \& kommunikation, Berlin.
} 
der Befragung teilgenommen, darunter $58 \%$, die auf Landesebene (Mitglied des Landtages oder Ministerium) tätig waren, $11 \%$ arbeiteten auf regionaler Ebene (Landräte, Kreistage) und $24 \%$ in Kommunen (Stadt- oder Gemeinderäte \& Bürgermeister).

Ohne frühe Öffentlichkeitsbeteiligung sind Infrastrukturprojekte kaum mehr möglich In einem ersten Schritt ging es um die Klärung, ob die am stärksten mit Bau- und Infrastrukturprojekten befassten Politiker die Notwendigkeit einer erweiterten und vorgezogenen Öffentlichkeitsbeteiligung grundsätzlich bestätigen können. Mehr als die Hälfte aller Befragten (53\%) glaubten hier, dass ohne frühe Öffentlichkeitbeteiligung große Infrastrukturprojekte eigentlich nicht mehr möglich sind; allerdings ist der Glaube an die Leistungsfähigkeit von Bürgerbeteiligungsverfahren auch begrenzt, denn für $60 \%$ der Befragten garantierte die frühe Öffentlichkeitsbeteiligung keinen reibungslosen Ablauf. Schaut man auch auf einzelne Kommentare, dann lassen sich neuralgische Punkte identifizieren: So wurde von einzelnen Teilnehmern hervorgehoben, wie entscheidend es ist, die Ergebnisse der Beteiligungsverfahren auch in das Projektmanagement zu überführen, respektive sie verbindlich zu machen. Auch wurde betont, dass sich starker Unmut und hohe Kosten vermeiden lassen.

Im Planungsprozess kann die frühe Öffentlichkeitsbeteiligung vor allem die Funktion erfüllen, Bürgern und Interessengruppen zu signalisieren, dass sie ernstgenommen werden $(63,2 \%)$ und dass Partizipation und Dialog gewünscht sind $(53,3 \%)$. Analytisch betrachtet liefert die frühe Öffentlichkeitsbeteiligung Hinweise darauf, was besonders kritisch betrachtet wird und damit Teil der Risikobewertung im Blick auf spätere Verfahrenshemmnisse oder Rechtsstreitigkeiten werden sollte. Auch kann sich der Planungsprozess dadurch insgesamt verbessern, glaubten 35,7\% der Berufspolitiker (siehe Tab. 1).

Beteiligungsverfahren können zu hohe Erwartungen wecken und Enttäuschung sogar verstärken Auffallend ist die Vielzahl von Kommentaren, die sich durch alle Fragen hindurchziehen und vor überzogenen Erwartungen an öffentliche Beteiligungsprozesse warnen. Das ist für uns eine der deutlichsten Überraschungen unter den Befragungsergebnissen. Wir hatten dazu keine direkte Frage gestellt, erhielten aber eine besondere Fülle von Einzelkommentaren. Denn zum einen ersetzen die Beteiligungsverfahren aus Sicht der Politiker ja schon aufgrund der fehlenden Legitimation nicht den öffentlichen Planungsprozess und entlasten auch das formelle Genehmigungsverfahren nur in geringem Umfang.

Zudem könne schnell der Eindruck entstehen, man dürfe wirklich mitentscheiden. Doch dazu müssten Beteiligungsverfahren nach Ansicht einiger 
Tab. 1 Welche Funktion erfüllt frühe Öffentlichkeitsbeteiligung? ( $\mathrm{n}=272$; Mehrfachnennungen möglich)

Frage: Welche Funktion kann frühe Öffentlichkeitsbeteiligung Ihrer Ansicht nach bestenfalls erfüllen?

\begin{tabular}{l|l|l}
\hline $\begin{array}{l}\text { Frühe Öffentlichkeitsbeteiligung schafft Vertrauen und zeigt dem Bürger } \\
\text { und Anspruchsgruppen, dass sie ernst genommen werden }\end{array}$ & 172 & $63,2 \%$ \\
\hline $\begin{array}{l}\text { Frühe Öffentlichkeitsbeteiligung ermöglicht aktive Partizipation durch } \\
\text { Dialog und Gesprächsbereitschaft }\end{array}$ & 145 & $53,3 \%$ \\
\hline $\begin{array}{l}\text { Frühe Öffentlichkeitsbeteiligung dient vor allem als Frühwarnsystem } \\
\text { und zur Risikominimierung (z. B. durch Verhinderung späterer Rechts- } \\
\text { streitigkeiten) }\end{array}$ & 114 & $41,9 \%$ \\
\hline $\begin{array}{l}\text { Frühe Öffentlichkeitsbeteiligung führt zu einer Verbesserung der } \\
\text { Planungsprozesse und Planungsergebnisse }\end{array}$ & 97 & $35,7 \%$ \\
\hline \begin{tabular}{l} 
Frühe Öffentlichkeitsbeteiligung entlastet die Genehmigungsverfahren \\
\hline $\begin{array}{l}\text { Frühe Öffentlichkeitsbeteiligung ist ein Steuerungsinstrument in der } \\
\text { offentlichen Diskussion }\end{array}$
\end{tabular} & 44 & $16,2 \%$ \\
\hline Nichts davon, sondern... & 11 & $34,6 \%$ \\
\hline
\end{tabular}

Befragter noch konkreter werden: „Frühe Öffentlichkeitsbeteiligung simuliert nur eine scheinbare Mitbestimmung. Diese muss durch neue Elemente erweitert werden, die eine verbindliche, basisdemokratische Partizipation ermöglichen", forderte ein Teilnehmer. Das werden zwar nicht alle so sehen, doch immer wieder kam zumindest die Forderung auf, es solle gleich am Anfang festgelegt werden, ob das Verfahren denn eher informativen oder tatsächlich partizipativen Charakter haben solle. Auch sahen einige Befragte die Gefahr einer Verzerrung der Ergebnisse durch Beteiligungsverfahren, da die Mehrheit der Bevölkerung sich am Ende doch nicht beteilige und somit Einzelinteressen von Aktivisten sich in den Verfahren durchsetzen würden, wie die Aussage eines Befragten deutlich macht: „Die Überbewertung öffentlich vorgetragener Einzelinteressen kann dazu führen, dass die schweigende Mehrheit faktisch entmachtet wird und die Mandatsträger einer nur scheinbaren Mehrheit folgen.“

\section{Bürgerbeteiligung zwischen Entscheidungsvorbereitung und Dauerauf-} gabe Deutsche Politiker sahen vor allem in der ersten Projektphase, in der am meisten Einfluss auf den Verlauf eines Projektes genommen werden kann, den größten Bedarf für öffentliche Beteiligung (41\%). Anders als bisher, wo im Rahmen von Genehmigungs- und Planfeststellungsverfahren vor allem über bereits 
vorliegende Pläne diskutiert wurde, wünschten sich die Befragten eine längere Diskussion über die grundlegenden Fragen, ,ob und, was ' überhaupt gebaut werden soll. Sie wollen den Bürger also an grundlegenden Fragen der Planung und Gestaltung stärker beteiligen als bisher. Für Großprojekte brauche es nach ihrer Ansicht einen breiten gesellschaftlichen Konsens.

Nach der ersten Phase der Planung sahen allerdings nur noch wenige Befragte einen Sinn in der Fortsetzung der Beteiligungsverfahren. Als Grund für diese Ablehnung wurde beispielsweise die Vorbeugung späterer (Jedermann-)Einsprüchen genannt. Knapp $15 \%$ der Befragten schlugen vor, lediglich bis zum Abschluss eines Genehmigungsverfahrens die Öffentlichkeit einzubeziehen. Auch Öffentlichkeitsbeteiligung im Umfeld oder kurz vor Beginn der Baumaßnahmen standen nicht hoch im Kurs. Hier lässt sich vermuten, dass Stakeholder-Management noch nicht als zentraler Managementprozess, sondern lediglich als punktuelle und zeitlich begrenzte Risikominimierung verstanden wird. Eine zweite Gruppe unter den deutschen Politikern beurteilte die Lage allerdings anders: Immerhin $36 \%$ der Befragten hielten öffentliche Beteiligungsverfahren für alle Phasen von Bau- und Infrastrukturprojekten für geboten - von der

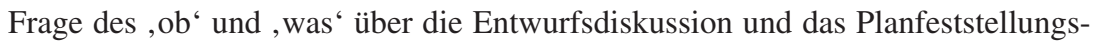
verfahren bis zur konkreten Durchführung.

Wer sollte für eine Beteiligung der Öffentlichkeit sorgen? Deutsche Politiker auf Landes-, Regional- und Kommunalebene sahen vor allem sich selbst (77,2 \%) und die jeweiligen Vorhabenträger $(70,3 \%)$ in der Verantwortung für die frühe und aktive Öffentlichkeitsbeteiligung. In Mecklenburg-Vorpommern wurden die Vorhabenträger bereits zu mehr Bürgerbeteiligung verpflichtet; andere Bundesländer dürften nachziehen. Doch auch genehmigende Behörden, beteiligte Bürger oder zivilgesellschaftliche Gruppen sahen die Befragten je nach Fall mit in der Pflicht (siehe Tab. 2). Einzelne Berufsgruppen - vertreten durch die jeweiligen Verbände - waren bislang überhaupt nicht im Fokus der deutschen Landes- und Regionalpolitiker. Infrastrukturprojekte werden als ,lokales' Ereignis zwischen Kommune, Vorhabenträger und Genehmigungsbehörden interpretiert. Einige glaubten sogar, dass mehr Bürgerbeteiligung nur im lokalen Kontext sinnvoll sei, aber nicht bei überregionalen oder übergeordneten Projekten, wo die Verantwortlichkeiten schwerer zu identifizieren sind.

Fragt man allerdings danach, ob die Branchenverbände sich stärker als bisher engagieren sollten, fällt das Bild differenzierter aus. Knapp 35\% der Befragten glaubten, dass eine stärkere Bekanntheit von früher Öffentlichkeitsbeteiligung erreicht werden könnte, wenn sich die Verbände stärker engagieren würden. $30 \%$ hielten dies hingegen für nicht notwendig, weitere $30 \%$ glaubten, die Verbände 
Tab. 2 Wer soll für eine frühe Öffentlichkeitsbeteiligung sorgen? $(n=232$; Mehrfachnennungen möglich)

Frage: Wen sehen Sie in der Rolle des Hauptverantwortlichen bei der Frühen Öffentlichkeitsbeteiligung im Falle der Bau- und Infrastrukturmaßnahmen?

\begin{tabular}{l|l|l}
\hline Den jeweiligen Vorhabenträger & 163 & $70,3 \%$ \\
\hline Die Kommunen bzw. die politisch verantwortlichen Instanzen & 179 & $77,2 \%$ \\
\hline Verbände und Vereinigungen (wie VDI, VCI, VDMA etc.) & 14 & $8,0 \%$ \\
\hline Die genehmigende Behörde & 55 & $23,7 \%$ \\
\hline Die beteiligten Bürger & 31 & $13,4 \%$ \\
\hline $\begin{array}{l}\text { Zivilgesellschaftliche Initiativen (wie z. B. Bürgerinitiativen, Kirchen, } \\
\text { NGOs) }\end{array}$ & 24 & $10,3 \%$ \\
\hline Sonstige, und zwar... & 6 & $2,6 \%$ \\
\hline
\end{tabular}

spielten dabei keine Rolle. Engagement wurde vor allem vom Bundesverband der Deutschen Industrie (BDI) als Spitzenverband $(23,8 \%)$ sowie von den bau-, energie- und ressourcennahen Verbänden Bundesverband der Energie- und Wasserwirtschaft (BDEW), Hauptverband der Bauindustrie, Verband beratender Ingenieure (VBI) und Bundesverband deutscher Wohnungs- und Immobilienunternehmen $(\mathrm{GdW})$ erwartet. Es dominierte - auch in den Einzelkommentaren das didaktische Ziel, Planern und Verantwortlichen die Bedeutung früher Öffentlichkeitsbeteiligung näherzubringen.

Neben den Industrieverbänden sahen die deutschen Politiker auch Umweltund Sozialverbände, Architektenkammern, Städte- und Gemeindetage und die Industrie- und Handelskammern in der Verantwortung, für mehr Beteiligung der Öffentlichkeit zu sorgen.

Die wichtigsten Stakeholder und Methoden: Vielfalt der Gruppen, Einfalt der Instrumente Während die befragten Politiker eine große Vielzahl relevanter Stakeholder bei Bau- und Infrastrukturmaßnahmen ausmachten, dominierten als wichtigste Instrumente der Beteiligung noch immer die klassische Bürgerinformationsveranstaltung $(87,4 \%)$ und die Entwurfspräsentation (51,6 \%). Ihnen gegenüber traten alle weiteren Instrumente wie Ständige Planungskonferenzen $(32,9 \%)$ oder Workshops zwischen Vorhabenträgern und Betroffenen $(41,3 \%)$ in den Hintergrund (siehe Tab. 3).

Viele dieser Maßnahmen, wie etwa der Einsatz von Szenariotechnik, wurden in den begleitenden Kommentaren als zu zeitaufwendig empfunden. Daher seien diese besonders komplexen oder krisenhaften Situationen vorbehalten. 
Tab. 3 Welches sind die wichtigsten Methoden und Instrumente der frühen Öffentlichkeitsbeteiligung? ( $\mathrm{n}=222$; Mehrfachnennungen möglich)

Frage: Was sind nach Ihrer Ansicht die richtigen Methoden oder Instrumente für die frühe Öffentlichkeitsbeteiligung?

\begin{tabular}{|c|c|c|}
\hline $\begin{array}{l}\text { Entwurfspräsentationen, öffentliche Auslage, Aushänge und Schaukästen } \\
\text { bei den zuständigen Behörden }\end{array}$ & 114 & $51,4 \%$ \\
\hline Öffentliche Bürgerinformationsveranstaltungen & 194 & $87,4 \%$ \\
\hline $\begin{array}{l}\text { Ständige Planungskonferenzen, die die Vorhaben begleiten und zwischen } \\
\text { den beteiligten Gruppen Kompromisse finden }\end{array}$ & 73 & $32,9 \%$ \\
\hline Workshops zwischen Vorhabenträgern und den Betroffenen & 92 & $41,3 \%$ \\
\hline $\begin{array}{l}\text { Regelmäßige Medienangebote wie Newsletter und kontinuierliche Presse- } \\
\text { arbeit }\end{array}$ & 99 & $44,6 \%$ \\
\hline $\begin{array}{l}\text { Dialogmedien wie Web 2.0, auf denen ständig neue Informationen } \\
\text { angeboten und Dialogangebote gemacht werden }\end{array}$ & 65 & $29,3 \%$ \\
\hline $\begin{array}{l}\text { Gründung von Projektbeiräten respektive Installation von Ombudsleuten } \\
\text { und Mediationen, die Partizipation sicherstellen und im Konfliktfall ver- } \\
\text { mitteln }\end{array}$ & 63 & $28,4 \%$ \\
\hline Bürgerentscheide & 32 & $14,4 \%$ \\
\hline $\begin{array}{l}\text { Szenarioplanung, in der auch Krisenszenarios durchgespielt und Krisen- } \\
\text { pläne entwickelt werden }\end{array}$ & 25 & $11,3 \%$ \\
\hline Sonstiges, und zwar... & 10 & $4,5 \%$ \\
\hline
\end{tabular}

Auch Methoden der E-Democracy wurden gegenwärtig noch keine besondere Bedeutung beigemessen: $37 \%$ standen elektronischen Verfahren der Meinungsund Willensbildung skeptisch gegenüber, $20 \%$ glaubten, dass diese Instrumente stets nur einen kleinen Teil der Bevölkerung ansprechen würden. Doch ein Blick auf die Breite der Stakeholder (zur Einschätzung der Relevanz verschiedener Stakeholder siehe Tab. 4) verrät, dass sich mit den gewählten Hauptmedien viele Gruppen nur unzureichend bedienen lassen.

Das trifft sowohl auf die relativ breite Streuung politischer Entscheider zu als auch auf die unterschiedlichen Kreise und Gruppen, in die sich die Betroffenen gliedern. Zusammen mit der Geringschätzung von Instrumenten wie der Szenarioplanung und den eher geringen Werten, die Dialogelemente und Entscheidungsplattformen erreichen, offenbart sich ein antiquiertes Stakeholderverständnis. Doch Stakeholder sind meist konkrete Gruppen, haben Namen, Interessen, konkrete Ziele und sind mit anderen Gruppen oder Individuen vernetzt (McVea und Freeman 2005). Für die Politik bedeutet das, sich weitaus stärker als 
Tab. 4 Welche Stakeholder sind Ihrer Meinung nach die wichtigsten bei Bau- und Infrastrukturmaßnahmen? $(\mathrm{n}=222$; Mehrfachnennungen möglich)

Frage: Welche Stakeholder sind Ihrer Meinung nach am wichtigsten bei Bau- und Infrastrukturmaßnahmen?

\begin{tabular}{l|l|l}
\hline Die Nachbaren/Anwohner, die von der Maßnahme betroffen sind & 160 & $72,1 \%$ \\
\hline $\begin{array}{l}\text { Die Parlamente und politischen Fraktionen auf kommunaler und } \\
\text { regionaler Ebene }\end{array}$ & 156 & $70,3 \%$ \\
\hline $\begin{array}{l}\text { Die Exekutive auf lokaler und regionaler Ebene, wie Bürgermeister, } \\
\text { Magistrate, Landräte etc. }\end{array}$ & 117 & $52,7 \%$ \\
\hline Bürgerinitiativen und Umweltschutzgruppen & 102 & $45,9 \%$ \\
\hline Die Vorhabenträger & 131 & $59,0 \%$ \\
\hline $\begin{array}{l}\text { Lokale Institutionen, Vereine, Gruppierungen, die sich betroffen zeigen } \\
\text { könnten }\end{array}$ & 79 & $35,6 \%$ \\
\hline Die betroffenen Unternehmen (und Konkurrenten) & 36 & $16,2 \%$ \\
\hline Genehmigungs- und Aufsichtsbehörden & 59 & $26,6 \%$ \\
\hline Wissenschaftler und Gutachter & 30 & $13,5 \%$ \\
\hline Medien und Presse & 90 & $40,5 \%$ \\
\hline Sonstige, und zwar... & 14 & $6,3 \%$ \\
\hline
\end{tabular}

bisher auf die einzelnen Stakeholder und ihren Interessen einzulassen. Daher sind für ein Projekt oder eine Klasse von Projekten spezifische und individuelle Stakeholderlandkarten zu entwickeln, sodass vorher eingeschätzt werden kann, was erfolgskritische Erwartungen sein werden.

Das trifft sich auch mit den Erwartungen der befragten Landes- und Regionalpolitiker: $37 \%$ glaubten, dass die Notwendigkeit, die Öffentlichkeit an solchen Verfahren zu beteiligen, stark steigen wird. Und $51 \%$ erwarteten, dass dies zu deutlichem Mehraufwand in der Projektplanung und Projektdurchführung führen wird.

\section{$5 \quad$ Fazit}

Zusammenfassend kann festgestellt werden, dass Politiker, die sich in Deutschland auf kommunaler, regionaler oder Länderebene mit partizipativen Elementen bei Bau- und Infrastrukturprojekten konfrontiert sehen, grundsätzlich offen für die Erwartungen der Bürger sind, sich aktiver an der Planung und Gestaltung 
solcher Projekte zu beteiligen. Allerdings fehlt ihnen dazu noch die geeignete Methodik. Auch die Gefahren eines solchen partizipativen Vorgehens sind den Befragten nur allzu präsent: Gerät ein großes Projekt in die Diskussion, wird das den Planungszeitraum vermutlich verlängern und das Projekt könnte sogar gänzlich scheitern. Deswegen empfehlen einige sogar, partizipative Elemente nur lokal begrenzt zur Anwendung zu bringen.

Dieses Ergebnis zeigt allerdings ein deutliches Unbehagen im Blick auf die Leistungsfähigkeit partizipativer Verfahren auf, das unter den Befragten vorherrscht. Ebenso wird ein gewisses Dilemma deutlich: Einerseits gehen die meisten befragten Politiker davon aus, dass partizipative Elemente gerade bei großen Vorhaben zunehmen werden. Andererseits kümmern sie sich zu wenig um Verfahren und Methoden, wie in einem Beteiligungsprozess durch das Einbeziehen möglichst vieler Positionen eine tragfähige Lösung gefunden werden kann. Damit gerät auch die grundlegende Fragestellung in den Blick, ob man Bürgerbeteiligung eben nur entscheidungsvorbereitend oder direktdemokratisch anlegen will. Die befragten Politiker sind hier unentschieden. Somit scheint ein Konflikt für die kommenden Jahre vorprogrammiert zu sein. Aus den Ergebnissen dieser Studie lassen sich sechs Thesen für die Zukunft der Öffentlichkeitsbeteiligung ableiten, die im Folgenden vorgestellt werden.

\section{Sechs Thesen zur Zukunft der Öffentlichkeitsbeteiligung}

1. Gestiegene Beteiligungsansprüche und veränderte Teilhabepräferenzen der Bevölkerung sind mehr als eine Modeerscheinung. Politik und Verwaltung werden sich langfristig auf sie einstellen müssen und sind aufgefordert, geeignete Beteiligungsverfahren zu erproben.

2. Die Entscheidung, ob eine Öffentlichkeitsbeteiligung entscheidungsbegleitend oder direktdemokratisch organisiert werden soll, lässt sich nicht umgehen. Die zentrale Frage lautet dabei, wann und warum welches Modell sinnvoll ist. Statt vorrangig auf eine neutrale Moderation zu setzen, sollten vielmehr auf der Grundlage eines gesamthaften Projekttableaus gemeinsam mit Befürwortern, Kritikern und Gegnern Ziele und Formate des Beteiligungsverfahrens festgelegt werden.

3. Frühe Öffentlichkeitsbeteiligung sollte Bestandteil jeder Planung von Bauund Infrastrukturmaßnahmen sein - und zwar fest verankert im gesamten Rahmen des technischen, finanziellen und genehmigungsrechtlichen Projektmanagements. 
4. Frühe Öffentlichkeitsbeteiligung kann in vielen Fällen die Planung verbessern und ermöglicht eine reibungslosere Umsetzung großer Vorhaben. Sie garantiert aber nicht, dass alle Betroffenen mit den Entscheidungen zufrieden sind.

5. Deutsche Kommunal- und Regionalpolitiker sind mit Blick auf Beteiligungsverfahren noch zögerlich und wenig experimentierfreudig. Wer dem Bürgerwunsch nach stärkerer Beteiligung aber nachkommen will, wird offener mit Beteiligungsformaten umgehen und finanzielle Ressourcen bereitstellen müssen.

6. Die empfundene Lücke zwischen legitimen Verwaltungsverfahren und legitimationsbedürftigen Beteiligungs- und Dialogformaten sollte geschlossen werden: Bürgerbeteiligungsverfahren können und sollen die gesetzlich vorgeschriebenen Verfahren nicht ersetzen, sie können aber mit ihnen verknüpft und aufeinander bezogen werden.

\section{Literatur}

Bertelsmann Stiftung (Hrsg.). (2014). Mehr Transparenz und Bürgerbeteiligung. Prozessschritte und Empfehlungen am Beispiel von Fernstraßen, Industrieanlagen und Kraftwerken. Gütersloh: Bertelsmann Stiftung.

Bundesministerium für Verkehr, Bau und Stadtentwicklung (Hrsg.). (2012). Handbuch für eine gute Bürgerbeteiligung. Planung von Großvorhaben im Verkehrssektor. Berlin: BMVI.

Crouch, C. (2008). Postdemokratie. Frankfurt a. M.: Suhrkamp.

Geißel, B., Roth, R., Collet, S., \& Tillmann, C. (2014). Partizipation und Demokratie im Wandel: Wie verändert sich unsere Demokratie durch neue Kombinationen repräsentativer, deliberativer und direktdemokratischer Elemente? In Bertelsmann Stiftung \& Staatsministerium Baden-Württemberg (Hrsg.), Partizipation im Wandel. Unsere Demokratie zwischen Wählen, Mitmachen und Entscheiden (S. 11-39). Gütersloh: Bertelsmann Stiftung.

Heller, S. (2018). Verhindern und Ermöglichen: Die Zunahme von Bürgerbeteiligung und die Folgen für das Funktionieren des demokratischen Systems. In N. Remus \& L. Rademacher (Hrsg.), Handbuch NGO-Kommunikation (S. 3-14). Wiesbaden: Springer VS.

Krappel, T., \& Freiherr von Süsskind-Schwendi, B. (2012). Der Entwurf eines „PlVereinhG“. Öffentlichkeitsbeteiligung, Verfahrensvereinheitlichung und Verfahrensbeschleunigung. UPR - Umwelt- und Planungsrecht, 32(7), 255-258.

Lintemeier, K., \& Rademacher, L. (Hrsg.). (2013). Stakeholder Relations. Nachhaltigkeit und Dialog als strategische Erfolgsfaktoren. München: Macromedia.

Luhmann, N. (1996). Die Realität der Massenmedien. Opladen: Westdeutscher Verlag.

McVea, J. F., \& Freeman, R. E. (2005). A names-and-faces approach to stakeholder management. How focusing on stakeholders as individuals can bring ethics and entrepreneurial strategy together. Journal of Management Inquiry, 14(1), 57-69.

Rademacher, L., \& Lintemeier, K. (2015). Sparte Partizipation?! Warum es noch kein Erfolgsmodell für Beteiligung und Dialog gibt. Berlin: DialogGesellschaft. 
Römmele, A., \& Schober, H. (Hrsg.). (2013). The governance of large-scale projects. Linking citizens and the state. Baden-Baden: Nomos.

RWE. (2012). Akzeptanz für Großprojekte. Eine Standortbestimmung über Chancen und Grenzen der Bürgerbeteiligung in Deutschland. Essen: RWE.

Schwab, J. (2014). Frühe Öffentlichkeitsbeteiligung und behördliche Genehmigungsverfahren. UPR - Umwelt- und Planungsrecht, 34(8), 281-290.

Staatsministerium Baden-Württemberg. (2014). Leitfaden für eine neue Planungskultur. http://mitwirkung.bw21.de/Downloads/140304_Planungsleitfaden.pdf. Zugegriffen: 1 . Juni 2019.

Verein Deutscher Ingenieure e. V. (2014). VDI 7000. Frühe Öffentlichkeitsbeteiligung bei Industrie- und Infrastrukturprojekten. https://www.vdi.de/richtlinien/details/ vdi-7000-fruehe-oeffentlichkeitsbeteiligung-bei-industrie-und-infrastrukturprojekten. Zugegriffen: 3. Juni 2019.

Wehrle, M. (2015). Befördert werden Ja-Sager. Zeit Online./Interviewer: Sabine Hockling. https://www.zeit.de/karriere/2015-09/nein-sagen-job-grenzen-setzen-kollegen-chef. Zugegriffen: 1. Juni 2019.

Open Access Dieses Kapitel wird unter der Creative Commons Namensnennung 4.0 International Lizenz (http://creativecommons.org/licenses/by/4.0/deed.de) veröffentlicht, welche die Nutzung, Vervielfältigung, Bearbeitung, Verbreitung und Wiedergabe in jeglichem Medium und Format erlaubt, sofern Sie den/die ursprünglichen Autor(en) und die Quelle ordnungsgemäß nennen, einen Link zur Creative Commons Lizenz beifügen und angeben, ob Änderungen vorgenommen wurden.

Die in diesem Kapitel enthaltenen Bilder und sonstiges Drittmaterial unterliegen ebenfalls der genannten Creative Commons Lizenz, sofern sich aus der Abbildungslegende nichts anderes ergibt. Sofern das betreffende Material nicht unter der genannten Creative Commons Lizenz steht und die betreffende Handlung nicht nach gesetzlichen Vorschriften erlaubt ist, ist für die oben aufgeführten Weiterverwendungen des Materials die Einwilligung des jeweiligen Rechteinhabers einzuholen.

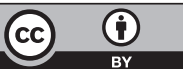

